\title{
UNIQUENESS AND DIFFERENTIAL CHARACTERIZATION OF APPROXIMATIONS FROM MANIFOLDS OF FUNCTIONS ${ }^{1}$
}

\author{
BY DANIEL WULBERT
}

Communicated by Victor Klee, July 27, 1970

Our purpose is to present the following result (definitions will be found below).

THEOREM. Let $M$ be a connected n-dimensional Haar embedded manifold in $C(K)$ whose restrictions, to any set of $n+1$ points $\left\{k_{i}\right\} \subseteq K$, form a closed hypersurface of $C\left(\left\{k_{i}\right\}\right)$. Then $M$ is a Chebyshev set.

The intention is to find sufficient conditions that a $C^{1}$ manifold is a Chebyshev set. We have intentionally not assumed several prevalent hypotheses from other nonlinear approximation theories such as, assuming that $K$ is a real interval, assuming a priori that $M$ is boundedly compact (this, however, will be found to be a consequence of our hypotheses) or explicitly assuming that

$$
\operatorname{card}\{k \in K: f(k)=g(k), f \text { and } g \text { in } M\} \leqq n .
$$

For example, such conditions arise in the unisolvent and locally unisolvent theories ([3], [6], and [5], see [5, 8.3] for a brief comparison of the nonlinear results of L. Tornheim, T. Motzkin, J. Rice, G. Meinardus and D. Schwedt). The possibility and desirability of approximating from manifolds was suggested in [8], where the uniqueness of approximation from manifolds in a smooth space is also discussed. Our setting is antipodal in the sense that it is fundamental to our argument that approximations from a Chebyshev subspace of $C(X)$ always satisfy a strong uniqueness condition [7]. The analogous statement in a smooth space is always false (Corollary $2 \mathrm{~b}$ below). Extensions of the necessity portion of Lemma 3 below have appeared in [2], [4], and [5, Theorem 89]. However it is the sufficiency of the characterization in Lemma 3 which is of primary

AMS 1969 subject classifications. Primary 4160, 4690; Secondary 4625, 4130, 4140, 4645 .

Key words and phrases. Nonlinear approximation, local best approximations, Chebyshev sets, strong uniqueness, Haar spaces, differential characterization of approximations, uniqueness of approximations.

1 This research was supported in part by a grant from the National Science Foundation.

Copyright @ 1971, American Mathematical Society 
utility in our development. Our definition of a Haar embedded manifold is a variation of a definition in [5, p. 142], and it follows for example that Theorems 91 and 92 in [5] can be obtained from Lemma 3.

We have outlined the proof by stating the major intermediate steps as lemmas, some of which are of independent interest. We have included a proof of the theorem from the lemmas. Although this is not the most difficult proof in the outline, it best illustrates the relation of the lemmas to the theorem. (Lemmas 1 and 5 are not explicitly referred to in the proof, but are used to prove Lemmas 3 and 6.) Complete proofs will appear elsewhere.

Definitions. If $K$ is a compact Hausdorff space, $C(K)$ denotes the real-valued continuous functions on $K$. Let $M$ be a manifold embedded in a Banach space $E$. Let $x$ be in $E$. Let

$$
d(x, M)=\inf \{\|x-m\|: m \text { in } M\} .
$$

A point $m$ in $M$ is termed a best approximation (local best approximation resp.) to $x$ (if there is a neighborhood $U$ of $m$ resp.) such that $\|m-x\|=d(x, M)(\|m-x\|=d(x, M \cap U)$ resp.). If for each $x$ in $E$ there is a unique best approximation to $x$ from $M$ then $M$ is a Chebyshev set. An $n$-dimensional linear subspace of $C(K)$ is a Haar space if zero is the only member vanishing on $n$-points. (It is classically known that a finite-dimensional subspace of $C(K)$ is a Chebyshev set if and only if it is a Haar space.) We identify the tangent space at a point $m$ in $M$ with a subspace $T(m)$ in $E$. If $E=C(K)$ and $M$ is an $n$-dimensional $C^{1}$-submanifold we say that $M$ is $H a a r$ embedded if $T(M)$ is an $n$-dimensional Haar space for each $m$ in $M$. Finally if $m$ is in $M$ and $x$ is in $E$ we say that $m$ satisfies a (local resp.) strong uniqueness condition for $x$ if there is an $r>0$ such that for all $z$ in $M$ (for all $z$ in some neighborhood of $m$, resp.)

$$
\|z-x\| \geqq\|m-x\|+r\|z-m\| .
$$

1. Lemma. Let 0 be a best approximation from a linear space $M$ to a point $y$ of norm one. The following are equivalent.

(i) There is an $r>0$ such that for all $p$ in $M$

$$
\sup \{L p:\|L\|=1=L y\} \geqq r\|p\| .
$$

(ii) 0 satisfies a strong uniqueness condition.

(iii) 0 satisfies a local strong uniqueness condition.

2. Corollary. (a) Approximations from a finite-dimensional Chebyshev subspace of an $L_{1}$-space, or of a $C_{\sigma}(K)$-space, always salisfy a strong uniqueness condition. 
(b) Approximations from a subspace of a smooth Banach space never satisfy a strong uniqueness condition.

In order to work with a single set of hypotheses, we will assume for the remainder of the results that $M$ is a connected $n$-dimensional Haar embedded manifold in $C(K)$. Let 0 be in $M$, and let $f$ be in $C(K)$.

3. LEMMA. 0 is a local best approximation to from $M$ if and only if 0 is a best approximation to from $T(0)$.

4. LEMMA. The following set valued mapping is lower semicontinuous

$$
P(f)=\{m \text { in } M: m \text { is a local best approximation to } f\} .
$$

5. LEMMA. If 0 is a best approximation to from $M$, and if $m$ is in $M$, then $m$ is a best approximation to $f+m$ from $M$.

6. Lemma. If card $K<\infty$, and $M$ is a closed hypersurface in $C(K)$, then 0 is a best approximation (global) to $f$ if and only if 0 is a best approximation to $f$ from $T(0)$.

Proof of THE TheOREM. We first show that best approximations are unique. Suppose that zero is a local best approximation to $f$. By a routine argument (using for example Lemma 3, the characterization for approximations from Haar spaces, and Lemma 3 again) there exists a set of $n+1$ points $\left\{x_{i}\right\}$ such that zero is a local best approximation to $f$ on $\left\{x_{i}\right\}$. By Lemma 6 , zero is a global best approximation to $f$ on $\left\{x_{i}\right\}$. Hence zero is a global best approximation on $K$. Hence since every local best approximation is a global best approximation, Lemma 4 implies that the set valued metric projection

$$
A(x)=\{m \text { in } M:\|m-x\|=d(x, M)\}
$$

is lower semicontinuous. This implies that $A(x)$ does not contain a proper subset which is compact and open relative to $A(x)$. (A proof can be constructed in the spirit of the proof for Theorem 14 in [1].) Since, by Lemma 3 and Corollary 2(a), each point in $A(x)$ is isolated, we conclude that $A(x)$ contains at most one point.

It remains to show that each point in $C(K)$ has a best approximation from $M$. We need to know that no two points in $M$ agree on $n+1$ points of $K$. Suppose that $m$ is a function in $M$ which agrees with zero on $n+1$ points. Let $L$ be a norm one annihilator of $T(0)$ which is supported on the $n+1$ zeros of $m$. Choose $f$ to be a continuous function such that $\|f\|=L(f)=1$. Thus by Lemma 6 and the characterizations for linear approximations, zero is a best approximation to $f$. Again using $L$, one shows that zero is a best approximation to $h$ $=(2\|m\|-|m|) f$. Some computation shows that $m$ is also a best 
approximation. This contradicts the first part of the proof.

We have that if $P$ is the restriction of functions in $M$ to any fixed set of $n+1$ points then $P$ is a regular differential mapping (since the tangent spaces are Haar spaces), and $P$ is one-to-one (by the last paragraph). An inverse function theorem argument implies that $M$ is homeomorphic to $P(M)$. It is now clear that $M$ is boundedly compact, and thus $M$ is a Chebyshev set. The proof is completed.

In the above proof we established several properties of Haar embedded manifolds. We record these properties in the next corollary.

7. Corollary. If $M$ satisfies the conditions of the theorem, then $M$ is a boundedly compact manifold which is diffeomorphic to $n$-space. Furthermore a point in $M$ which is a local best approximation to a function $f$ is, in fact, the unique global best approximation to $f$.

8. EXAMPLE. Let

$$
M=\{(r \cos t, r \sin t, t+r \sin (t+\pi / 4)): t \text { real and } r>\sqrt{2}\} .
$$

Then $M$ is a 2 -dimensional Haar embedded manifold in $R^{3}$. However there exist points which have nonunique best approximations from $M$. Hence we cannot simply drop the closure assumption in the theorem and still prove uniqueness for approximations. The computations necessary for the example are facilitated by an appropriate use of Lemma 3.

\section{REFERENCES}

1. J. Blatter, P. D. Morris and D. E. Wulbert, Continuity of the set-valued metric projection, Math. Ann. 178 (1968), 12-24. MR 37 \#4563.

2. R. Bonic, Points of minimum norm on smooth surfaces in Banach spaces, Proc. Amer. Math. Soc. 18 (1967), 1004-1005. MR 37 \#57.

3. P. C. Curtis, Jr., n-parameter families and best approximation, Pacific J. Math. 9 (1959), 1013-1027. MR 21 \#7385.

4. T. M. Flett, Points of minimum or maximum norm on smooth surfaces in Banach spaces, J. London Math. Soc. 44 (1969), 583-586. MR 39 \#1969.

5. G. Meinardus, Approximation of functions: Theory and numerical methods, Springer, Berlin, 1964; English transl., Springer Tracts in Natural Philosophy, vol. 13, Springer-Verlag, New York, 1967. MR 31 \#547; MR 36 \#571.

6. T. S. Motzkin, Approximation by curves of a unisolvent family, Bull. Amer. Math. Soc. 55 (1949), 789-793. MR 11, 101.

7. D. J. Newman and H. S. Shapiro, Some theorems on Čebyšev approximation, Duke Math. J. 30 (1963), 673-681. MR 27 \#6070.

8. J. R. Rice, Non-linear approximation, Proc. Sympos. Approximation of Functions (General Motors Res. Lab., 1964), Elsevier, Amsterdam, 1965, pp. 111-133. MR 33 \#6229.

9. D. E. Wulbert, Uniqueness and differential characterization of approximations from manifolds of functions, Amer. J. Math. (to appear).

University of Washington, Seattle, Washington 98105 